\title{
Coloring Geographical Threshold Graphs
}

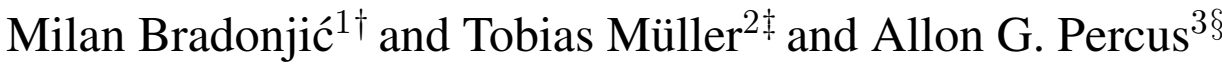 \\ ${ }^{1}$ Theoretical Division, and Center for Nonlinear Studies, Los Alamos National Laboratory, Los Alamos, USA. \\ ${ }^{2}$ School of Mathematics, Tel Aviv University, Israel. \\ ${ }^{3}$ School of Mathematical Sciences, Claremont Graduate University, Claremont, USA.
}

received $3^{\text {rd }}$ September 2009, accepted $22^{\text {nd }}$ June 2010.

\begin{abstract}
We propose a coloring algorithm for sparse random graphs generated by the geographical threshold graph (GTG) model, a generalization of random geometric graphs (RGG). In a GTG, nodes are distributed in a Euclidean space, and edges are assigned according to a threshold function involving the distance between nodes as well as randomly chosen node weights. The motivation for analyzing this model is that many real networks (e.g., wireless networks, the Internet, etc.) need to be studied by using a "richer" stochastic model (which in this case includes both a distance between nodes and weights on the nodes). Here, we analyze the GTG coloring algorithm together with the graph's clique number, showing formally that in spite of the differences in structure between GTG and RGG, the asymptotic behavior of the chromatic number is identical: $\chi=\frac{\ln n}{\ln \ln n}(1+o(1))$. Finally, we consider the leading corrections to this expression, again using the coloring algorithm and clique number to provide bounds on the chromatic number. We show that the gap between the lower and upper bound is within $C \ln n /(\ln \ln n)^{2}$, and specify the constant $C$.
\end{abstract}

Keywords: Geographical threshold graphs, random geometric graphs, chromatic number, coloring algorithm.

\section{Introduction}

Numerous approaches have been proposed in recent years to study the structure of large real-world technological and social networks, and to optimize processes on these networks. A particularly fertile approach has been to consider the network as an instance of an ensemble, arising from a suitable random generative model. One straightforward example is the random geometric graph (RGG) model, where nodes are placed at random in a Euclidean space and edges are placed between any two nodes within a threshold distance. This has the advantage of describing many aspects of systems such as sensor networks, while avoiding unnecessary detail. Even though geometric correlations in RGGs complicate the probabilistic

\footnotetext{
${ }^{\dagger}$ Email: milan@lanl.gov. Supported by the Department of Energy ASCR program at Los Alamos National Laboratory under contract DE-AC52-06NA25396.

$\ddagger$ Current address: Centrum Wiskunde \& Informatica, P.O. Box 94079, 1090GB Amsterdam, The Netherlands. Email: tobias@cwi.nl. Partially supported through an ERC advanced grant.

Email: allon.percusecgu.edu. Supported by National Science Foundation grant CCF-0829945 and Office of Naval Research grant N000141010641.
}

1365-8050 (c) 2010 Discrete Mathematics and Theoretical Computer Science (DMTCS), Nancy, France 
analysis of the model, recent work has clarified many of its structural properties including threshold behavior Penrose (2003); Gupta and Kumar (1998); Goel et al. (2004), random walk behavior Avin and Ercal (2007) and chromatic number McDiarmid and Müller; Müller (2008); Penrose (2003).

RGGs fail, however, to capture heterogeneity in the network. Geographical threshold graphs (GTG) aim at generalizing RGGs, providing this heterogeneity via a richer stochastic model that nevertheless preserves much of the simplicity of the RGG model. GTGs assign to nodes both a location and a weight, which may represent a quantity such as transmission power in a wireless network or influence in a social network. Edges are placed between two nodes if a symmetric function of their weights and the distance between them exceeds a certain threshold Bradonjić and Kong (2007).

Recent work has analyzed structural properties of GTGs, such as connectivity, clustering coefficient, degree distribution, diameter, existence and absence of the giant component Bradonjić et al. (2007, 2008). These properties are not merely of theoretical importance, but also play an important role in applications. In communication networks, connectivity implies the ability to reach all parts of the network. In packet routing, the diameter gives the minimal number of hops needed for transmission between two arbitrary nodes. And in the case of epidemics, the existence or absence of the giant component controls whether the epidemic spreads or is contained.

When considering wireless networks, a natural quantity to study is the chromatic number. This is the minimum number of colors needed to color vertices, such that no two adjacent vertices in the graph receive the same color. Treating the colors as the different radio channels or frequencies, the chromatic number gives the minimal number of channels needed so that neighboring radios do not interfere with each other. In this paper we study the asymptotic behavior of the chromatic number for GTGs with constant mean degree. We propose a greedy coloring algorithm, and analyze the behavior of this algorithm along with the graph's clique number. This leads to upper and lower bounds on the chromatic number.

The paper is organized as follows. Section 2 defines the GTG model. Section 3 presents our main asymptotic result, based on our analysis of the coloring algorithm. We show that for graphs $G$ of constant mean degree, both the clique number $\omega(G)$ and chromatic number $\chi(G)$ are with high probability $\frac{\ln n}{\ln \ln n}(1+o(1))$. Section 4 analyzes the gap between lower and upper bounds on the chromatic number, given respectively by the clique number and the greedy coloring algorithm. We show that this gap is at most $C \ln n /(\ln \ln n)^{2}$, and bound the constant $C$. Finally, Section 5 concludes with open questions regarding the chromatic number for sparser and denser GTGs.

\section{Geographical Threshold Graph Model}

Given random points $X_{1}, X_{2}, \cdots \in[0,1]^{2}$ that are i.i.d., uniformly at random, and i.i.d. nonnegative weights $W_{1}, W_{2}, \ldots$, we construct a random geographical threshold graph $G_{n}$ as follows. Let $N \stackrel{d}{=} \operatorname{Po}(n)$ be the number of nodes, independent of the $X_{i}$ and $W_{i}$. Let $\theta_{n}$ be a given threshold parameter that depends on $n$. Then $G_{n}$ has vertex set $V\left(G_{n}\right)=\{1, \ldots, N\}$, and for $i, j \in V\left(G_{n}\right), G_{n}$ has edge $i j \in E\left(G_{n}\right)$ iff

$$
\frac{W_{i}+W_{j}}{\left\|X_{i}-X_{j}\right\|^{2}} \geq \theta_{n}
$$

For technical convenience we identify opposite edges of $[0,1]^{2}$, making it into a torus.

We will specifically analyze the regime of constant expected degree. If $\mathbf{E}\left(W_{i}\right)$ is a constant, then this occurs when the threshold parameter is linear in the expected number of nodes, $\theta_{n}=\Theta(n)$. For 
simplicity we take $\theta_{n}=n$, since if $\theta_{n}=c n$ for some constant $c>0$, the weights can always be rescaled to $W_{i}:=W_{i} / c$.

\section{Asymptotic Results}

If $G$ is a graph then $\omega(G)$ denotes its clique number and $\chi(G)$ its chromatic number. We will show formally that the clique number and chromatic number of the geographical threshold graph are essentially the same as those for a random geometric graph with the same (constant) average degree.

Note that, since coloring a clique of size $\omega(G)$ requires $\omega(G)$ different colors, $\omega(G) \leq \chi(G)$.

Theorem 3.1 Suppose that weights are distributed such that $\operatorname{Pr}\left(W_{i}>x\right)=O\left(x^{-\gamma}\right)$ for some $\gamma>1$. Then

and

$$
\frac{\omega\left(G_{n}\right)}{\ln n / \ln \ln n} \rightarrow 1 \quad \text { in probability, }
$$

$$
\frac{\chi\left(G_{n}\right)}{\ln n / \ln \ln n} \rightarrow 1 \quad \text { in probability, }
$$

as $n \rightarrow \infty$.

The rest of this section is devoted to proving the theorem.

\subsection{Lower bound}

Let $\hat{w} \in \mathbf{R}$ be such that $\operatorname{Pr}\left(W_{i}>\hat{w}\right) \geq 1 / 2$. Then the probability that $G_{n}$ contains fewer than $n / 3$ vertices with weight more than $\hat{w}$ is exponentially small. In fact, this probability is bounded above by the probability that a $\operatorname{Po}(n / 2)$-variable is less than $n / 3$, which is $\exp (-\Omega(n))$, as can be seen by the Chernoff bound. Let $G_{n}^{\prime}$ be the subgraph of $G_{n}$ induced by $n / 3$ of the points with weight at least $\hat{w}$. Note that if $i, j \in V\left(G_{n}^{\prime}\right)$ and $\left\|X_{i}-X_{j}\right\|^{2}<2 \hat{w} / n$ then certainly $i j \in E\left(G_{n}^{\prime}\right)$. Thus $G_{n}^{\prime}$ (and hence also $G_{n}$ ) contains the ordinary random geometric graph $\operatorname{RGG}(n / 3, \sqrt{2 \hat{w} / n})$ as a subgraph, with probability $1-\exp (-\Omega(n))$. By Lemma 5.3 of McDiarmid 2003),

$$
\operatorname{Pr}\left(\omega\left(G_{n}\right)<(1-\varepsilon) \ln n / \ln \ln n\right)=o(1) .
$$

\subsection{Upper bound}

Let us define a "level" $L_{k}$ as follows:

$$
L_{k}:= \begin{cases}\left\{i \leq N: W_{i}<1\right\}, & k=-1 \\ \left\{i \leq N: 4^{k} \leq W_{i}<4^{k+1}\right\}, & k \geq 0 .\end{cases}
$$

Note that the set $\left\{X_{i}: i \in L_{k}\right\}$ of the points of the Poisson process corresponding to level $k$ (for $k \geq 0$ ) is in fact a Poisson process itself with intensity $n\left(F\left(4^{k+1}\right)-F\left(4^{k}\right)\right)$ on the unit square and intensity 0 elsewhere, where $F$ denotes the cdf of $W_{1}$. Moreover, these Poisson processes corresponding to the levels $L_{-1}, L_{0}, L_{1}, \ldots$ are independent.

For $x \in[0,1]^{2}$ let us denote

$$
M_{x}:=\sum_{k=-1}^{\infty}\left|\left\{i \in L_{k}:\left\|X_{i}-x\right\| \leq 6 \sqrt{2} \cdot 2^{k} / \sqrt{n}\right\}\right|,
$$


and let us set

$$
M:=\max _{x \in[0,1]^{2}} M_{x}
$$

Very roughly, $M$ represents the greatest number of neighbors that a sufficiently high-weight node can have. Then we have the following lemma.

Lemma 3.2 The chromatic number satisfies $\chi\left(G_{n}\right) \leq M$.

Proof: Let us order the vertices by nondecreasing weight and greedily color them. That is, we first color the vertex with smallest weight, then the vertex with second smallest weight and so on; and when we choose a color for a vertex we always pick the smallest available color (i.e., the smallest color that does not occur among the neighbors of the vertex that have already been colored). We claim that in this way we will never need more than $M$ colors.

For ease of notation let us assume (w.l.o.g.) that $W_{1} \leq W_{2} \leq \cdots \leq W_{N}$. Let $N_{<}(i)$ represent all neighbors of node $i$ with lower weight than $i$ :

$$
N_{<}(i):=\left\{j<i: i j \in E\left(G_{n}\right)\right\} .
$$

Note that if $i \in L_{k}$ and $j \in N_{<}(i)$ then $\left\|X_{i}-X_{j}\right\| \leq 2^{k+3 / 2} / \sqrt{n}$. For $1 \leq i \leq N$ let $c(i)$ denote the color that the algorithm has assigned to vertex $i$. Observe that for each $1 \leq i \leq N$ we have

$$
\{1, \ldots, c(i)-1\} \subseteq\left\{c(j): j \in N_{<}(i)\right\},
$$

because otherwise the algorithm would not have used color $c(i)$ for $i$.

Now let $i$ be an arbitrary vertex. For notational convenience let us write $j_{0}:=i$ and let $k_{0}$ denote the level of $i$. Let $m$ be the maximal number for which there exist strictly decreasing sequences $j_{0}>j_{1}>$ $\cdots>j_{m}$ and $k_{0}>k_{1}>\cdots>k_{m}$ with the following properties. For each $p=0, \ldots, m-1$ we have

1. $c\left(j_{p+1}\right)=\max \left(\left\{1, \ldots, c\left(j_{p}\right)-1\right\} \backslash\left\{c(j): j \in N_{<}\left(j_{p}\right) \cap L_{k_{p}}\right\}\right)$;

2. $j_{p+1} \in L_{k_{p+1}} \cap N_{<}\left(j_{p}\right)$.

(Observe that, for each color $c \in\left\{1, \ldots, c\left(j_{p}\right)-1\right\} \backslash\left\{c(j): j \in N_{<}\left(j_{p}\right) \cap L_{k_{p}}\right\}$ - provided this set is non-empty - there is a $j \in N_{<}\left(j_{p}\right)$ with $c(j)=c$ by (2) and the level of $j$ is necessarily strictly less than $k_{p}$.) It is possible that $m=0$. In that case $\left\{1, \ldots, c\left(j_{0}\right)-1\right\} \subseteq\left\{c(j): L_{k_{0}} \cap N_{<}\left(j_{0}\right)\right\}$. This gives

$$
\begin{aligned}
c(i) & \leq\left|L_{k_{0}} \cap N_{<}(i)\right|+1 \\
& \leq\left|\left\{j \in L_{k_{0}}:\left\|X_{j}-X_{i}\right\| \leq 2^{k_{0}+3 / 2} / \sqrt{n}\right\}\right| \\
& \leq M_{X_{i}} \\
& \leq M .
\end{aligned}
$$

Now suppose that $m>0$. By maximality of $m$ we have

$$
\left\{1, \ldots, c\left(j_{m}\right)-1\right\} \subseteq\left\{c(j): L_{k_{m}} \cap N_{<}\left(j_{m}\right)\right\} .
$$

Also note that, by definition of $j_{p}$ we have

$$
\left\{c\left(j_{p+1}\right)+1, \ldots, c\left(j_{p}\right)-1\right\} \subseteq\left\{c(j): j \in L_{k_{p}} \cap N_{<}\left(j_{p}\right)\right\} \quad \text { for } p=0, \ldots, m-1 .
$$


Combining (3) and (4) we find

$$
\begin{aligned}
c(i) & =\left|\left\{1, \ldots, c\left(j_{m}\right)-1\right\}\right|+1+\sum_{p=0}^{m-1}\left|\left\{c\left(j_{p+1}\right)+1, \ldots, c\left(j_{p}\right)-1\right\}\right|+1 \\
& \leq \sum_{p=0}^{m}\left|L_{k_{p}} \cap N_{<}\left(j_{p}\right)\right|+m+1 \\
& \leq \sum_{p=0}^{m}\left|\left\{j \in L_{k_{p}}:\left\|X_{j}-X_{j_{p}}\right\| \leq 2^{k_{p}+\frac{3}{2}} / \sqrt{n}\right\}\right| .
\end{aligned}
$$

Let us observe that:

$$
\begin{aligned}
\left\|X_{j_{p}}-X_{j_{m}}\right\| & \leq \sum_{q=p}^{m-1}\left\|X_{j_{q}}-X_{j_{q+1}}\right\| \leq \sum_{q=p}^{m-1} 2^{k_{q}+3 / 2} / \sqrt{n} \\
& <2^{k_{p}+5 / 2} / \sqrt{n}
\end{aligned}
$$

for all $1 \leq p \leq m$. Thus, if $\left\|X_{j}-X_{j_{p}}\right\|<2^{k_{p}+\frac{3}{2}} / \sqrt{n}$ then

$$
\begin{aligned}
\left\|X_{j}-X_{j_{m}}\right\| & \leq\left\|X_{j_{p}}-X_{j_{m}}\right\|+\left\|X_{j}-X_{j_{p}}\right\| \\
& \leq\left(2^{k_{p}+5 / 2}+2^{k_{p}+3 / 2}\right) / \sqrt{n} \\
& =6 \sqrt{2} \cdot 2^{k_{p}} / \sqrt{n} .
\end{aligned}
$$

Combining (6) with (5) we get:

$$
\begin{aligned}
c(i) & \leq \sum_{p=0}^{m}\left|\left\{j \in L_{k_{p}}:\left\|X_{j}-X_{j_{m}}\right\| \leq 6 \sqrt{2} \cdot 2^{k_{p}} / \sqrt{n}\right\}\right| . \\
& \leq M_{X_{j_{m}}} \\
& \leq M .
\end{aligned}
$$

Since the vertex $i$ was arbitrary, the claim follows.

Remark: Note that Lemma 3.2 does not depend on the fact that the points and weights are random, but rather it will hold for any "deterministic" threshold graph if we replace $n$ by its threshold $\theta_{n}$ in the definition of $M_{x}$ above.

To finish the proof of the theorem it now suffices to prove the following lemma.

Lemma 3.3 Suppose that weights are distributed such that $\operatorname{Pr}\left(W_{i}>x\right)=O\left(x^{-\gamma}\right)$ for some $\gamma>1$. Then, for any $\varepsilon>0$ :

$$
\operatorname{Pr}\left(M \geq(1+\varepsilon) \frac{\ln n}{\ln \ln n}\right) \rightarrow 0,
$$

as $n \rightarrow \infty$.

Proof: Let us set

$$
M_{x}^{\prime}:=\sum_{k=-1}^{\infty}\left|\left\{i \in L_{k}:\left\|X_{i}-x\right\| \leq 12 \sqrt{2} \cdot 2^{k} / \sqrt{n}\right\}\right|,
$$

and $A:=\left\{\left(\frac{a}{\sqrt{n}}, \frac{b}{\sqrt{n}}\right): 0 \leq a, b \leq \sqrt{n}\right\}$ (where $a$ and $b$ are integers). Now, for any $x \in[0,1]^{2}$ there is $z \in A$ with $\|x-z\| \leq \sqrt{2} / \sqrt{n}$. So if $\left\|X_{i}-x\right\| \leq 6 \sqrt{2} \cdot 2^{k} / \sqrt{n}$ then $\left\|X_{i}-z\right\| \leq \sqrt{2} / \sqrt{n}+6 \sqrt{2} \cdot 2^{k} / \sqrt{n}<$ $12 \sqrt{2} \cdot 2^{k} / \sqrt{n}$ for all $k \geq-1$, and thus

$$
M \leq \max _{x \in A} M_{x}^{\prime} .
$$


Let $x \in \mathbf{R}^{2}$ be arbitrary and note that $M_{x}^{\prime} \stackrel{d}{=} \sum_{k=-1}^{\infty} Z_{k}$, where the $Z_{k}$ are independent Poisson random variables, and

$$
\begin{aligned}
\mathbf{E}\left(Z_{k}\right) & = \begin{cases}\pi(12 \sqrt{2})^{2} 4^{-1} F(1), & k=-1 \\
\pi(12 \sqrt{2})^{2} 4^{k}\left(F\left(4^{k+1}\right)-F\left(4^{k}\right)\right), & k \geq 0 .\end{cases} \\
& =O\left(4^{k(1-\gamma)}\right) .
\end{aligned}
$$

So in particular $M_{x}^{\prime}$ is itself Poisson with a mean that is bounded above by some constant, say $\mu$. Using a well known bound, see for instance the Lemma 4.4 of McDiarmid (2003), we obtain that

$$
\begin{aligned}
\operatorname{Pr}\left(M_{x}^{\prime}>(1+\varepsilon) \ln n / \ln \ln n\right) & \leq\left(\frac{e \mu}{(1+\varepsilon) \ln n / \ln \ln n}\right)^{(1+\varepsilon) \ln n / \ln \ln n} \\
& =\exp (-(1+\varepsilon+o(1)) \ln n) .
\end{aligned}
$$

Hence, by Eq. (7), applying the union bound,

$$
\begin{aligned}
\operatorname{Pr}(M>(1+\varepsilon) \ln n / \ln \ln n) & \leq n e^{-(1+\varepsilon+o(1)) \ln n} \\
& \leq n^{-\frac{\varepsilon}{2}} .
\end{aligned}
$$

The last inequality holds for $n$ sufficiently large. This shows that $M /(\ln n / \ln \ln n)$ is upper bounded by $1+\varepsilon$, with high probability.

Remark: It is possible to adapt a subsequence trick from Penrose (2003) (page 123) to strengthen the type of convergence in Theorem 3.1 from convergence in probability to almost sure convergence. We now briefly sketch the main ideas.

The probability that the clique number of the RGG is less than $(1-\varepsilon) \ln n / \ln \ln n$ has been upper bounded by $\exp \left(-n^{\varepsilon+o(1)}\right)$ in McDiarmid (2003) (page 201). These computations combined with the Borel-Cantelli Lemma give that $\lim \inf M /(\ln n / \ln \ln n) \geq 1$ a.s.

To prove that $\lim \sup M /(\ln n / \ln \ln n) \leq 1$ a.s. let us define a sequence $\left(m_{k}\right)_{k}$ by setting $m_{k}:=k^{3 / \varepsilon}$. Then the upper bound 9 ) together with the Borel-Cantelli lemma give that

$$
\operatorname{Pr}\left(\frac{M\left(m_{k}\right)}{\ln m_{k} / \ln \ln m_{k}} \leq 1+\varepsilon \text { for all but finitely many } k\right)=1 .
$$

If instead of $\theta_{n}=\sqrt{n}$ we had taken $\theta_{n}^{(\varepsilon)}:=(1-\varepsilon) \sqrt{n}$ then all computations leading up to (10) would have held. For $n \in \mathbf{N}$, let $m(n):=\left\lceil n^{\varepsilon / 3}\right\rceil^{3 / \varepsilon}$ be the least element of $\left(m_{k}\right)_{k}$ with $n \leq m_{k}$. Observe that $\lim _{n \rightarrow \infty} m(n) / n=1$, so that in particular $\theta_{m(n)}^{(\varepsilon)} \leq \theta_{n}$ for all sufficiently large $n$. Now, let $M^{(\varepsilon)}$ denote the analogue of $M$, which we obtain if we use the same points $X_{1}, X_{1}, \ldots$ and weights $W_{1}, W_{2}, \ldots$, with the threshold $\theta_{n}^{(\varepsilon)}$ instead. Let us assume that the random variables $N(1), N(2), \ldots$, which determine the number of points used in the construction of the GTG and definition of $M$, have been coupled in such a way that $N(k) \leq N(k+1)$ for all $k$. (This can be achieved by setting $N(k)=Y_{1}+\cdots+Y_{k}$ with the $Y_{i}$ 's i.i.d. $\operatorname{Po}(1)$-variables). Then we obtain

$$
M(n) \leq M^{(\varepsilon)}(m(n)),
$$

for all sufficiently large $n$ (using that $\theta_{m(n)}^{(\varepsilon)} \leq \theta_{n}$ for all sufficiently large $n$ ). Combining [11] with (10) proves that $\lim \sup M /(\ln n / \ln \ln n) \leq 1$ a.s. 


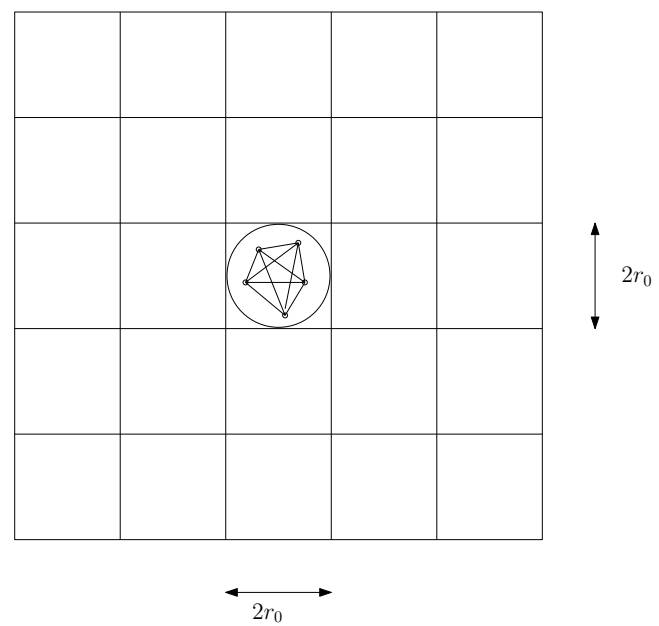

Fig. 1: Tiling of the unit square, showing one ball $B_{i}$ and the high-weight nodes $\left(W \geq w_{0}\right)$ within the ball.

\section{Mind the Gap!}

In this section we analyze the gap between lower and upper bounds on the chromatic number, given respectively by the clique number (Subsection 4.1) and the greedy coloring algorithm (Subsection 4.2). In Subsection 4.3 we show that this gap is at most $C \ln n /(\ln \ln n)^{2}$, and bound the constant $C$.

\subsection{Lower Bound}

Informally: we tile the space $[0,1]^{2}$ and inscribe a ball in each tile. Then the number of nodes within a clique in an arbitrarily chosen ball will give us a lower bound on the chromatic number of the entire geographical threshold graph within $[0,1]^{2}$. The number of the balls, or how we tile the space $[0,1]^{2}$, is a parameter that we discuss later. Formally, the argument is the following.

For some threshold weight $w_{0}$, let $\alpha$ be defined by $\operatorname{Pr}\left(W \leq w_{0}\right)=\alpha$. We will appropriately choose the constant $w_{0}$ (and hence $\alpha$ ) later. Let us define a radius $r_{0}=\sqrt{w_{0} /\left(2 \theta_{n}\right)}$. We consider $b=1 /\left(2 r_{0}\right)^{2}$ disjoint balls with radii $r_{0}$ (see Figure 1 ) and call these balls $B_{i}$. For convenience, tile the square $[0,1]^{2}$ with $b=1 /\left(2 r_{0}\right)^{2}$ sub-squares of the size $2 r_{0} \times 2 r_{0}$, and within each of the squares inscribe a ball of radius $r_{0}$. The number of nodes within $B_{i}$ is given by Poisson distribution $\operatorname{Po}\left(n r_{0}^{2} \pi\right)$, while the number of nodes with weights $\geq w_{0}$ within $B_{i}$ is given by $\operatorname{Po}\left((1-\alpha) n r_{0}^{2} \pi\right)$. For convenience we let $\lambda=$ $(1-\alpha) n r_{0}^{2} \pi$. Let us note that for $\theta_{n}=n$ it follows $b=1 /\left(4 r_{0}^{2}\right)=\theta_{n} /\left(2 w_{0}\right)=n /\left(2 w_{0}\right)$ (this is $\Theta(n)$ ) and $\lambda=\frac{\pi}{2}(1-\alpha) w_{0}$ (this is $\Theta(1)$ ).

Let us now consider only those nodes with weights $W \geq w_{0}$ within a given ball $B_{i}$. All such nodes form a clique, since by construction, each pair of nodes within $B_{i}$ satisfies the connectivity relation Eq. 11). Let $k$ be a positive integer to be specified later. Since the number of nodes $n_{i}$ within $B_{i}$ is a Poisson random variable with mean $\lambda$,

$$
\operatorname{Pr}\left(n_{i} \geq k\right) \geq e^{-\lambda} \frac{\lambda^{k}}{k !}
$$

Denote $p:=e^{-\lambda} \lambda^{k} / k$ !, and let $I_{i}$ be an indicator of the event $\left\{n_{i} \geq k\right\}$, so that $\operatorname{Pr}\left(I_{i}=1\right) \geq p$. Let us 
define $J=\sum_{i=1}^{b} I_{i}$. We will show that for sufficiently large $k, \operatorname{Pr}(J=0) \rightarrow 0$. First, $J=0$ iff all $I_{i}$ are 0 . Second, the indicators $I_{i}$ are mutually independent, since the balls $B_{i}$ are mutually disjoint. Thus, $\operatorname{Pr}\left(\cap I_{i}^{c}\right)=\operatorname{Pr}\left(I_{i}^{c}\right)^{b} \leq(1-p)^{b}=\exp (\ln (1-p) b)$. We have seen that $b=\Theta(n)$. Now choose $k$ so that $p=\ln n / n$. In that case, $\operatorname{Pr}(J>0) \geq 1-\exp (\ln (1-p) b)=1-\exp (-\Theta(\ln n))=1-n^{-\Theta(1)}$. Thus, we must solve the following equation in $k$

Taking the logarithm,

$$
e^{-\lambda} \frac{\lambda^{k}}{k !}=\frac{\ln n}{n}
$$

$$
\lambda-k \ln \lambda+\ln k !=\ln n-\ln \ln n .
$$

According to Stirling's formula $k !=\sqrt{2 \pi k} k^{k} e^{-k+\beta / 12 k}$ for some $\beta \in(0,1)$, and applying the logarithm, $\ln k !=\frac{1}{2} \ln 2 \pi k+k(\ln k-1)+\beta / 12 k$. Now, Eq. 14 is equivalent to

$$
k(\ln k-1-\ln \lambda)=\ln n-\ln \ln n-\lambda-\frac{1}{2} \ln 2 \pi-\frac{1}{2} \ln k-\frac{\beta}{12 k} .
$$

Let $\eta=1+\ln \lambda$ (this is $\Theta(1))$, and introduce the (rescaled) variables $y=e^{-\eta} k$ and $x=e^{-\eta}(\ln n-$ $\left.\ln \ln n-\lambda-\frac{1}{2} \ln 2 \pi-\beta / 12 k-\eta / 2\right)$. Then, Eq. 15 may be recast as

$$
y=\frac{x}{\ln y}-\frac{e^{-\eta}}{2} .
$$

For given $x$ and $\eta$, Eq. 16 has a unique solution in $y$. Noting that $x=\Theta(\ln n)$, it is not hard to verify that $y$ satisfies

$$
y=\frac{x}{\ln x}\left(1+\frac{\ln \ln x}{\ln x}(1+o(1))\right) .
$$

From the precise definition of $x$,

$$
x=e^{-\eta} \ln n\left(1-O\left(\frac{\ln \ln n}{\ln n}\right)\right),
$$

so

$$
\begin{aligned}
\ln x & =\ln \left(e^{-\eta} \ln n(1-o(1))\right) \\
& =\ln \ln n-\eta-o(1)
\end{aligned}
$$

and

$$
\begin{aligned}
\ln \ln x & =\ln (\ln \ln n(1-o(1))) \\
& =\ln \ln \ln n-o(1) .
\end{aligned}
$$

Therefore from Eq. (17),

$$
\begin{aligned}
\ln y & =\ln x-\ln \ln x+o(1) \\
& =\ln \ln n-\ln \ln \ln n-\eta-o(1) \\
& =\ln \ln n\left(1-\frac{\ln \ln \ln n+\eta+o(1)}{\ln \ln n}\right) .
\end{aligned}
$$


Plugging Eq. 18 and Eq. 19 , into the right-hand side of Eq. (16) gives

$$
\begin{aligned}
y & =\frac{e^{-\eta} \ln n\left(1-O\left(\frac{\ln \ln n}{\ln n}\right)\right)}{\ln \ln n\left(1-\frac{\ln \ln \ln n+\eta+o(1)}{\ln \ln n}\right)}-\frac{e^{-\eta}}{2} \\
& =\frac{e^{-\eta} \ln n}{\ln \ln n}\left(1+\frac{\ln \ln \ln n+\eta+o(1)}{\ln \ln n}\right)-\frac{e^{-\eta}}{2},
\end{aligned}
$$

so

$$
\begin{aligned}
k & =e^{\eta} y \\
& =\frac{\ln n}{\ln \ln n}\left(1+\frac{\ln \ln \ln n+\eta+o(1)}{\ln \ln n}\right) .
\end{aligned}
$$

We know that there is a clique of size at least $k$ within some ball $B_{i}$, with probability $\geq 1-n^{-\Theta(1)}$. Since $k \leq \omega\left(G_{n}\right) \leq \chi\left(G_{n}\right)$, it follows that with probability $\geq 1-n^{-\Theta(1)}$, the chromatic number satisfies

\subsection{Upper Bound}

$$
\frac{\ln n}{\ln \ln n}\left(1+\frac{\ln \ln \ln n+\eta+o(1)}{\ln \ln n}\right) \leq \chi\left(G_{n}\right) .
$$

In this subsection we derive an upper bound on the chromatic number, given by the greedy coloring algorithm in Section 3. Consider the inequality [8. Letting $B=1+\ln \mu$,

$$
\begin{aligned}
& \operatorname{Pr}\left(M_{x}^{\prime}>(1+\varepsilon) \ln n / \ln \ln n\right)=\left(\frac{e \mu}{(1+\varepsilon) \ln n / \ln \ln n}\right)^{(1+\varepsilon) \ln n / \ln \ln n} \\
&=\exp \left\{\left(B-\ln \left((1+\varepsilon) \frac{\ln n}{\ln \ln n}\right)\right)(1+\varepsilon) \frac{\ln n}{\ln \ln n}\right\} \\
&=\exp \left\{\ln n\left(\frac{B(1+\varepsilon)}{\ln \ln n}-(1+\varepsilon) \frac{\ln (1+\varepsilon)+\ln \ln n-\ln \ln \ln n}{\ln \ln n}\right)\right\} \\
&=\exp \left\{\operatorname { l n } n \left(\frac{B(1+\varepsilon)}{\ln \ln n}-\frac{(1+\varepsilon) \ln (1+\varepsilon)}{\ln \ln n}\right.\right. \\
&\left.\left.-(1+\varepsilon)+(1+\varepsilon) \frac{\ln \ln \ln n}{\ln \ln n}\right)\right\} \\
&=\exp \left\{\operatorname { l n } n \left(\frac{B}{\ln \ln n}+\frac{B \varepsilon}{\ln \ln n}-\frac{\varepsilon+O\left(\varepsilon^{2}\right)}{\ln \ln n}\right.\right. \\
&\left.\left.-1-\varepsilon+\frac{\ln \ln \ln n}{\ln \ln n}+\varepsilon \frac{\ln n}{\ln \ln n}\right)\right\} .
\end{aligned}
$$

Let us choose $\varepsilon$ to be

$$
\varepsilon=\frac{\ln \ln \ln n+B+\delta}{\ln \ln n}
$$

for some $\delta$. Then it follows that

$$
\begin{aligned}
\operatorname{Pr}\left(M_{x}^{\prime}>(1+\varepsilon) \ln n / \ln \ln n\right) & \leq \exp \left\{\ln n\left(-1-\frac{\delta}{\ln \ln n}+\varepsilon \frac{\ln \ln \ln n+B-1-o(1)}{\ln \ln n}\right)\right\} \\
& =\exp \left\{\ln n\left(-1-\frac{\delta-o(1)}{\ln \ln n}\right)\right\}
\end{aligned}
$$


Hence, by Eq. (7) and the union bound, $\operatorname{Pr}(M<(1+\varepsilon) \ln n / \ln \ln n) \geq 1-e^{-\frac{\ln n}{\ln \ln n}(\delta-o(1))}$. Consequently, for any positive constant $\delta$, the chromatic number satisfies

$$
\chi\left(G_{n}\right) \leq \frac{\ln n}{\ln \ln n}\left(1+\frac{\ln \ln \ln n+B+\delta}{\ln \ln n}\right)
$$

with probability $\geq 1-\exp (-\Theta(\ln n / \ln \ln n))$.

\subsection{Comparison of Bounds}

Let us now optimize the constants $\eta=1+\ln \lambda$ and $B=1+\ln \mu$ to minimize the gap between lower and upper bounds on $\chi\left(G_{n}\right)$. We define $s_{1}=\sup _{\alpha \in[0,1]} \eta$ and $s_{2}=\inf _{\alpha \in[0,1]} B$. By using the definition of $\alpha=\operatorname{Pr}\left(W \leq w_{0}\right)$, we obtain

$$
\begin{aligned}
s_{1} & =1+\sup _{\alpha \in[0,1]} \ln \lambda \\
& =1+\sup _{\alpha \in[0,1]} \ln \left((1-\alpha) n r_{0}^{2} \pi\right) \\
& =1+\sup _{\alpha \in[0,1]} \ln \left(\frac{\pi}{2} \frac{n}{\theta_{n}}(1-\alpha) w_{0}\right) \\
& =1+\ln \frac{\pi}{2}+\sup _{w_{0} \geq 0} \ln \left(\left(1-F\left(w_{0}\right)\right) w_{0}\right) \\
& =1+\ln \frac{\pi}{2}+\ln \left(\sup _{w_{0} \geq 0} w_{0}\left(1-F\left(w_{0}\right)\right)\right) .
\end{aligned}
$$

For the other bound, $s_{2}=1+\inf _{\alpha \in[0,1]} \ln \mu$, and $\mu$ is bounded by

$$
\begin{aligned}
\mu & \leq \sum_{j=-1}^{\infty} \mathbf{E}\left(Z_{j}\right) \\
& =\pi(12 \sqrt{2})^{2}\left(4^{-1} F(1)+\sum_{j=0}^{\infty} 4^{j}\left(F\left(4^{j+1}\right)-F\left(4^{j}\right)\right)\right) \\
& =\pi(12 \sqrt{2})^{2}\left(\frac{1}{4}+\frac{3}{4} \sum_{j=0}^{\infty} 4^{j}\left(1-F\left(4^{j}\right)\right)\right)
\end{aligned}
$$

so

$$
s_{2} \leq 1+\ln \left(72 \pi\left(1+3 \sum_{j=0}^{\infty} 4^{j}\left(1-F\left(4^{j}\right)\right)\right)\right) .
$$

Note that the conditions imposed on the weight distribution in Lemma $3.3 \operatorname{are} \operatorname{Pr}\left(W_{i}>x\right)=O\left(x^{-\gamma}\right)$ for some $\gamma>1$. Then it may be helpful to write $1-F\left(4^{j}\right)=O\left(4^{-\gamma j}\right) \leq D 4^{-\gamma j}$, with the constant $D$ given by $D=\max _{j} 4^{\gamma j}\left(1-F\left(4^{j}\right)\right)$. In that case,

$$
\begin{aligned}
s_{2} & \leq 1+\ln \left(72 \pi\left(1+3 \sum_{j=0}^{\infty} 4^{j} D 4^{-\gamma j}\right)\right) \\
& =1+\ln \left(72 \pi\left(1+3 D \sum_{j=0}^{\infty} 4^{(1-\gamma) j}\right)\right) \\
& =1+\ln \left(72 \pi\left(1+\frac{3 D}{1-4^{1-\gamma}}\right)\right) .
\end{aligned}
$$


Now the lower and upper bounds on $\chi\left(G_{n}\right)$, respectively,

$$
\frac{\ln n}{\ln \ln n}\left(1+\frac{\ln \ln \ln n+s_{1}}{\ln \ln n}\right) \leq \chi\left(G_{n}\right)
$$

and

$$
\chi\left(G_{n}\right) \leq \frac{\ln n}{\ln \ln n}\left(1+\frac{\ln \ln \ln n+s_{2}}{\ln \ln n}\right)
$$

give us the size of the gap $C \ln n /(\ln \ln n)^{2}$.

Finally, the constant $C$, specified in the abstract, is $C=s_{2}-s_{1}$, where $s_{1}$ and $s_{2}$ are as above.

\subsection{Examples of Bounds}

Here we compare lower and upper bounds for the following two weight distributions: (i) exponential and (ii) power-law.

(i) Exponential weight distribution: $f(w)=e^{-w}$, for $w \geq 0$, and thus $F(w)=1-e^{-w}$.

Since $\sup _{w_{0} \geq 0} w_{0}\left(1-F\left(w_{0}\right)\right)=1 / e$ is attained at $w_{0}=1$, then Eq. 20p yields

$$
s_{1}=\ln (\pi / 2) \approx 0.4516
$$

On the other hand, Eq. 21] yields

$$
s_{2} \leq 1+\ln \left(72 \pi\left(1+3 \sum_{j=0}^{\infty} 4^{j} \exp \left(-4^{j}\right)\right)\right) \approx 7.2644,
$$

giving $C<6.82$.

(ii) Power-law weight distribution: $f(w)=w^{-\beta}$, for $w \geq 1$, and thus $F(w)=1-1 / w^{\beta-1}$.

Since $\sup _{w_{0} \geq 1} w_{0}\left(1-F\left(w_{0}\right)\right)=\sup _{w_{0} \geq 1} 1 / w_{0}^{\beta-2}=1$, attained at $w_{0}=1$ and for $\beta \geq 2$, then Eq. 20] yields

$$
s_{1}=1+\ln (\pi / 2) \approx 1.4516,
$$

while Eq. 21 becomes

$$
\begin{aligned}
s_{2} & \leq 1+\ln \left(72 \pi\left(1+3 \sum_{j=0}^{\infty} 4^{j(2-\beta)}\right)\right) \\
& =1+\ln \left(72 \pi\left(1+\frac{3}{1-4^{2-\beta}}\right)\right) \\
& \approx 6.4214+\ln \left(1+\frac{3}{1-4^{2-\beta}}\right) .
\end{aligned}
$$

If for instance $\beta=3$, this last bound is $\approx 8.0308$, giving $C<6.58$. 


\section{Conclusion}

In this work, we have derived the chromatic number and proposed a coloring algorithm on GTG, for the case of $\theta_{n}=\Theta(n)$, that is, when the mean degree is constant. We are naturally interested into the values of the chromatic number for denser and sparser GTGs. A particularly interesting case would be to show the chromatic number around the connectivity regime. The connectivity threshold for GTGs has been derived to be $\theta_{n}=\Theta(n / \ln n)$, Bradonjić et al. (2007). However, the methods that we have used in this work rely heavily on the techniques that apply for RGGs of equivalent degree and the analysis of the chromatic number of the RGG is much more involved in this range than in the range we have studied in this work. For instance, in McDiarmid and Müller it has been shown that if the expected degree of the RGG is $t \ln n$ for some constant $t>0$, then the ratio of the chromatic number to the clique number has a limit, which can be expressed as some complicated function of $t$. One of the surprising findings of McDiarmid and Müller has been the existence of a constant $t_{0}>0$ such that this ratio tends to 1 for expected degrees $\leq t_{0} \ln n$ and it is strictly bigger than one if the expected degree is at least $\left(t_{0}+\varepsilon\right) \ln n$, for any $\varepsilon>0$.

It is unlikely that the crude upper bound on the chromatic number, which we have used in this work (that is, our $M$ ), would be anywhere near sharp when the expected degree is logarithmic. Therefore, we expect that substantial new tools will be needed to tackle the chromatic number of the GTG in this regime.

\section{References}

C. Avin and G. Ercal. On the cover time and mixing time of random geometric graphs. Theor. Comput. Sci., 380 (1-2):2-22, 2007.

M. Bradonjić and J. Kong. Wireless ad hoc networks with tunable topology. In Proceedings of the 45th Annual Allerton Conference on Communication, Control and Computing, 2007.

M. Bradonjić, A. A. Hagberg, and A. G. Percus. Giant component and connectivity in geographical threshold graphs. In Proceedings of the 5th Workshop on Algorithms and Models for the Web-Graph (WAW2007), pages 209-216, 2007.

M. Bradonjić, A. A. Hagberg, and A. G. Percus. The structure of geographical threshold graphs. Internet Mathematics, 5(1-2):113-139, 2008.

A. Goel, S. Rai, and B. Krishnamachari. Sharp thresholds for monotone properties in random geometric graphs. In STOC '04: Proceedings of the thirty-sixth annual ACM symposium on Theory of computing, pages 580-586, New York, NY, USA, 2004. ACM Press.

P. Gupta and P. R. Kumar. Critical power for asymptotic connectivity. In Proceedings of the 37th IEEE Conference on Decision and Control, volume 1, pages 1106-1110, 1998.

C. McDiarmid. Random channel assignment in the plane. Random Struct. Algorithms, 22(2):187-212, 2003.

C. McDiarmid and T. Müller. On the chromatic number of random geometric graphs. Combinatorica. To appear.

T. Müller. Two-point concentration in random geometric graphs. Combinatorica, 28(5):529-545, 2008.

M. D. Penrose. Random Geometric Graphs. Oxford University Press, 2003. 